\title{
Contrôle strict de la glycémie : quel futur ?
}

\section{Strict glycemic control: what does the future hold?}

\author{
J.-C. Preiser \\ (C) SRLF et Springer-Verlag France 2011
}

La mariée était-elle trop belle ? La vague d'enthousiasme soulevée en 2001 par la publication de Van den Berghe et al. [1], qui démontrait que l'insulinothérapie intensive titrée en vue d'atteindre une glycémie entre 4,4 et $6,1 \mathrm{mmol} / 1$ est retombée après l'échec de sept autres essais cliniques prospectifs randomisés indépendants (plus de 11000 patients inclus au total) [2]. La concordance des échecs de confirmation doit certainement nous inciter à la réflexion, après le déchaînement des passions qui a mené à des attitudes extrêmes : certains ont considéré que le contrôle strict de la glycémie était souhaitable à condition qu'il soit pratiqué par des mains expertes et motivées, alors que d'autres ont considéré qu'il fallait le jeter aux oubliettes. Ces attitudes diamétralement opposées sont aussi reflétées par les recommandations publiées ! Jugez plutôt : après la recommandation du contrôle strict de la glycémie en 2004 par le groupe des endocrinologues et diabétologues américains [3], l'abandon du contrôle glycémique est maintenant recommandé [4]. Plus modéré, un groupe de travail composé d'intensivistes et de réanimateurs sous les auspices de la Société de réanimation de langue française (SRLF) et de la Société française d'anesthésie et de réanimation (Sfar) recommande maintenant un contrôle modéré de la glycémie, c'est-à-dire une limite supérieure acceptable de $10 \mathrm{mmol} / \mathrm{l}$ [5] ; et en pratique courante, une limite supérieure de $8 \mathrm{mmol} / \mathrm{l}$ est souvent utilisée [6].

Ces errements dans les recommandations, comme le tumulte des passions, sont en fait révélateurs de nos incertitudes ; le concept même de départ, à savoir le bénéfice de la restauration d'une « normoglycémie » chez le patient agressé, doit être remis en question. La fourchette de glycémie de 4,4 à $6,1 \mathrm{mmol} / 1$ est considérée comme normale chez un sujet ambulatoire et à jeun, pour qui une intolérance glucidique est suspectée en cas de glycémie supérieure à $6,1 \mathrm{mmol} / \mathrm{l}$. Sur quelle base rationnelle cette même fourchette devraitelle être considérée comme « normale » chez le patient

J.-C. Preiser $(\bowtie)$

Service des soins intensifs, hôpital universitaire Erasme,

808, route de Lennik, B-1070 Bruxelles, Belgique

e-mail : Jean-Charles.Preiser@erasme.ulb.ac.be agressé soumis à un stress métabolique intense et le plus souvent alimenté ? [7].

La première question qui vient à l'esprit est donc : quelle est la glycémie souhaitable chez le patient de réanimation? La réponse ne tiendra probablement pas à un seul chiffre ou à une seule fourchette de valeurs, compte tenu de l'extrême variété des situations cliniques, des antécédents, du temps écoulé depuis l'agression, des traitements, etc. Cette question fondamentale et rémanente ne pourra être probablement résolue que par l'analyse de sous-groupes définis de patients, chez qui on étudiera les effets de l'obtention durable d'une cible glycémique.

La deuxième question est le corollaire de la première : comment atteindre rapidement et durablement une cible glycémique préétablie ? Ici, intervient une série d'éléments liés au patient, mais aussi techniques et organisationnels : quel dispositif de mesure de glucose ? Quelle fréquence de mesure ? Au prix de quelle charge de travail ? Quel algorithme thérapeutique?

La troisième question est celle de la nature de la cible glycémique : valeur glycémique moyenne ou variabilité glycémique ? Si l'atteinte d'une fourchette cible représente un objectif thérapeutique raisonnable avec nos moyens actuels, la minimisation de la variabilité nécessitera des moyens de mesure de contrôle glycémique beaucoup plus rapprochés.

Enfin, la quatrième question est celle de la prévention de l'hypoglycémie, cruciale en raison des risques qui lui sont associés, même si elle est modérée [8].

Heureusement, les outils nécessaires pour nous fournir les réponses à ces questionnements quasi existentiels ont été développés grâce à de fructueuses collaborations multidisciplinaires :

- la mesure continue de la glycémie par différentes techniques (enzymatique, spectroscopie à infrarouge, microdialyse, fibre optique) intravasculaire ou sous-cutanée a fait récemment l'objet des premiers tests cliniques et devrait bientôt nous aider à la recherche clinique en réanimation ;

- les algorithmes de contrôle glycémique par adaptation de la vitesse d'administration de l'insuline et/ou des apports 
glucidiques s'améliorent constamment, notamment grâce à l'adaptation des algorithmes mathématiques à la sensibilité à l'insuline. L'étude CGAO-Réa, actuellement en cours en France, devrait permettre de valider la qualité de cette approche ;

- les systèmes de contrôleurs par boucle fermée ou semiouverte (l'homme dans la boucle) devraient représenter l'aboutissement ultime de la recherche sur les algorithmes et pourraient alléger la charge de travail des soignants, en garantissant le maintien de la glycémie dans une cible choisie.

Enfin, des moyens thérapeutiques destinés aux diabétiques (insulines semi-lentes ou à longue durée d'action, agents insulinosensibilisateurs ou inhibiteurs de la dégradation des incrétines) font l'objet d'autres investigations dont les résultats pourraient également modifier les modalités de contrôle glycémique du malade agressé.

En conclusion, les champs d'investigation dans le domaine du contrôle glycémique de l'agressé sont immenses, à la mesure de l'enthousiasme initial et de l'importance des questions révélées. Pour la pratique quotidienne, gardons à l'esprit le précepte Primum non nocere de notre arrièregrand-père commun, Hippocrate, en évitant à nos patients les risques inconsidérés liés d'un contrôle glycémique « trop » strict.

\section{Références}

1. Van den Berghe G, Wouters P, Weekers F, et al (2001) Intensive insulin therapy in the critically ill patients. $\mathrm{N}$ Engl $\mathrm{J}$ Med 345:1359-67

2. Marik PE, Preiser JC (2010) Toward understanding tight glycemic control in the ICU: a systematic review and metaanalysis. Chest 137:544-51

3. Garber AJ, Moghissi ES, Bransome ED Jr, et al (2004) American College of Endocrinology position statement on inpatient diabetes and metabolic control. Endocr Pract 10:77-82

4. Qaseem A, Humphrey LL, Chou R, et al (2011) Use of intensive insulin therapy for the management of glycemic control in hospitalized patients: a clinical practice guideline from the American College of Physicians. Ann Intern Med 154:260-7

5. Ichai C, Preiser JC (2010) International recommendations for glucose control in adult non diabetic critically ill patients. Crit Care 14:R166

6. Krinsley JS, Preiser JC (2008) Moving beyond tight glucose control to safe effective glucose control. Crit Care 12:149

7. Preiser JC (2008) Restoring normoglycemia: not so harmless. Crit Care 12:116

8. Egi M, Bellomo R, Stachowski E, et al (2010) Hypoglycemia and outcome in critically ill patients. Mayo Clin Proc 85:217-24 